\title{
In-Situ Process Monitoring in Additive Manufacturing Using Statistics and Pre-Process Data
}

\author{
Eva Maria Scheideler*, Andrea Huxol
}

\begin{abstract}
Long computation times are a major obstacle for the application of in-situ monitoring in additive manufacturing. This paper presents rapid in-situ monitoring, which returns a control value within typical build times. Observing powder bed fusion processes reveals that unsuitable parameter settings influence the appearance of the molten surface and the surrounding powder bed. The presented research approach evaluates the changing appearance of the exposed layers, in combination with the information from the preprocess about the position and geometry of the components in each layer. Grayscale images are captured with the build envelope camera and examined regarding the grayscale distribution in the critical areas surrounding the component boundaries. The grayscale distribution is then used to predict product quality by using standard statistical methods. The combination of the pre-process data and the fast analysis of the grayscale distribution allows promptly calculating a performance indicator for required process intervention and control.
\end{abstract}

Keywords: energy density; image processing; layer check; part quality; Selective Laser Melting

\section{INTRODUCTION}

In many industries, additive manufacturing (AM) is increasingly focused on the production of final parts, as it has the potential to solve certain problems in the context of decreasing lot sizes and product individualization [1]. To exploit the full potential of the technology, AM has to be completely integrated into the production process. This requires certain changes in the production process itself. For instance, AM machines have to be embedded into industrial process chains by increasing the level of automation and developing consistent data models [2].

Furthermore, the process quality of AM processes has to be improved to allow the production of reliable part quality. The implementation of process monitoring systems can help to improve the limited or unproven repeatability of the build process. At the current state of research and in industrial applications, many AM machines are already equipped with certain process monitoring tools. Though the existing process monitoring solutions show a potential to improve the reliability of the SLM process, they are not yet fully applicable for process control [3].

\section{SELECTIVE LASER MELTING}

The term additive manufacturing (AM) describes different technologies that create parts directly from threedimensional CAD data, by additive joining of volume elements, usually in the form of layers [4]. Today, several AM technologies show a level of maturity that enables the application in series production. One of these is Laser Powder Bed Fusion of Metals (M-LPBF). In principle with this method, a layer of powder is spread onto a build platform and afterward selectively fused by use of a laser beam in the area where the part is to be generated. Next, the platform is lowered minimally and a new layer is spread and fused again. This procedure is repeated until the final height of the product is reached. Thus, the product is generated layer by layer, surrounded by the residual powder.
A large number of parameters and factors influences the outcome of the M-LPBF process, during the in-process as well as during pre- and post-processes. For the in-process, these parameters include the definition of the exposure strategy, e.g. hatch definition, laser energy and scan speed, but also the environmental control of the build chamber, including gas flow, atmosphere and temperature. The extensive number of influencing parameters hinders the development of suitable methods for process control and quality assurance, especially as the quantitative correlation between the parameters, the process signatures and the process results are mostly unknown [5]. Thus, quality control measures for M-LPBF are largely limited to quality inspection.

Current quality management (QM) techniques in traditional manufacturing are based on statistical analysis and require a large number of identical tests to create a sufficient database. As AM technologies are often applied for the production of small lot sizes, the process will not deliver an appropriate number of parts to apply these methods. Together with the limited availability of design rules and specified tolerance classes for $\mathrm{AM}$ technologies, it is extremely difficult to achieve a proven process capability [6]. The implementation of process monitoring systems in M-LPBF can help to evaluate and improve the repeatability of the build process. In the first stage, it can enable the identification of defects during the build process, in a final stage this may lead to the development of closed-loop control systems [7].

Various approaches towards process monitoring for additive manufacturing are presented in research, mostly using optical measurement methods for different indicators. For several of these approaches, it is shown, that the measured signals change in areas, where parts show defects. Nevertheless, full proof of applicability for inline process monitoring is not yet available [3].

In commercial M-LPBF machines, different process monitoring solutions are integrated. These systems can be divided into five categories: condition monitoring, powder bed monitoring, laser power monitoring, melt pool monitoring and documentation of the individual layer 
surfaces. Condition monitoring systems use a variety of sensors to collect data on the general operation of the machine and supervise safety-relevant parameters. All other categories of monitoring systems are based on optical measurements. Photo diodes are used for monitoring the laser power and certain emissions from the melt pool. For monitoring of larger areas, like in powder bed or layer surface monitoring as well as monitoring the size and shape of the melt pool, camera systems are applied.

At the current state, these systems are mainly collecting data, which is manually evaluated afterward. Changes in the signals are used as indicators for final part inspection. For applications of M-LPBF in series production, it is possible to compare the data of a larger number of parts to come to threshold values. However, this is not feasible for individual parts or very small lot sizes. Here, it is necessary to find a possibility to define general thresholds [3].

Furthermore, the large amount of data that is collected by the process monitoring tools is difficult to handle. Current systems mostly collect the data during the build process and analyse the entire data after the process is completed.

\section{RESEARCH APPROACH}

Currently, a lot of experience is required to operate an M-LPBF machine and produce high-quality parts. The experience gained by the operator can enable the identification of certain problems during the build process. For example, it is possible to identify parts with a very high energy input that have a high risk of curling or forming an uneven surface. This experience can be mimicked by image processing solutions. The presented research approach aims to develop an image processing methodology applicable to identify parts that are likely to show curling due to high local energy input. The methodology is intended to use simplified models for the calculation to achieve short processing times and thus, enable the application for in-process monitoring and control. This article provides the description of a practical implementation and solutions from the area of production.

\subsection{Preconditions}

An experimental design is prepared to identify influencing parameters on the part porosity. Therefore, cubes of $8 \times 8 \times 8 \mathrm{~mm}^{3}$ are built from a CoCrW dental alloy. The machine used in this experiment is a Realizer SLM 125 with a build chamber of $125 \times 125 \times 200 \mathrm{~mm}^{3}$. For documentation purpose, the machine captures images of each layer after spreading the powder layer and after the melting process by a monochromatic digital camera with a resolution of $1280 \times 720$ pixel.

The layer thickness is kept constant at $25 \mu \mathrm{m}$ and the hatch is changing between X-hatch in odd layers and Y-hatch in even layers. All parts are built with different parameter combinations resulting in different volumetric energy densities. The volumetric energy density $E$ is calculated according to Eq. (1)

$$
E=\frac{P}{\frac{d_{\mathrm{p}}}{t_{\mathrm{e}}} \cdot d_{1} \cdot d_{\mathrm{h}}}
$$

Where $P$ is the laser power in Watt, $d_{\mathrm{p}}$ is the point distance in $\mathrm{mm}, t_{\mathrm{e}}$ is the exposure time in $s, d_{1}$ is the layer thickness in $\mathrm{mm}$ and $\mathrm{d}_{\mathrm{h}}$ is the hatch distance in $\mathrm{mm}$. The parameters laser power, point distance and exposure time are varied between three different values each, which results in volumetric energy densities between 30.00 and $185.33 \mathrm{~J} / \mathrm{mm}^{3}$. The observation of the build jobs reveals irregularities for the samples with the highest energy densities, as shown in Fig. 1.

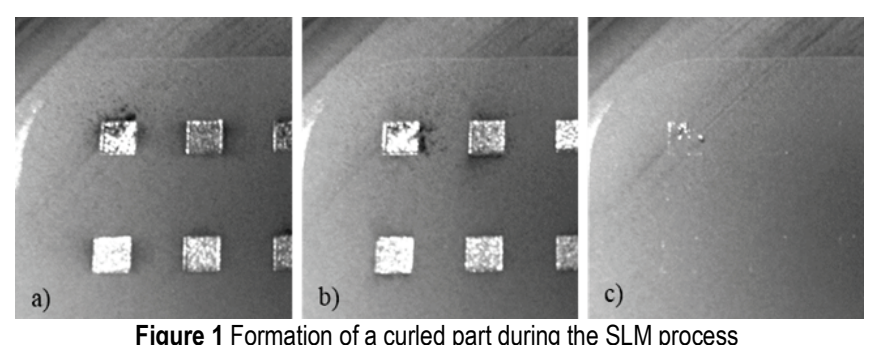

a) part height $0.65 \mathrm{~mm}, \mathrm{~b}$ ) part height $0.675 \mathrm{~mm}, \mathrm{c}$ ) powder layer at part height $1.125 \mathrm{~mm} .[8]$

For the top left sample in the pictures, an accumulation of burnt material is visible. Its position depends on the hatch direction (Fig. 1a and Fig. 1b). In successive layers a curling effect of the surface becomes obvious (Fig. 1c)) that can cause damages on the powder spreading system. These parts have to be deleted from the further build process.

\subsection{Image Processing}

The aim of the research approach is to develop an image processing methodology that can be applied to identify parts that are likely to show curling due to high local energy input. The methodology is intended to use simplified models for the calculation to achieve short processing times and thus, enable the application for in-process monitoring and control.

In a first approach, the images captured after the melting of the individual layers are used to identify areas where burnt material is accumulating near parts with high energy input. To identify differences between the good and the substandard parts, images from the experimental setup as described in 3.1 are analysed. Therefore, the surrounding areas of the cubic sample are separated into North, East, South and West regions, as shown in Fig. 2.

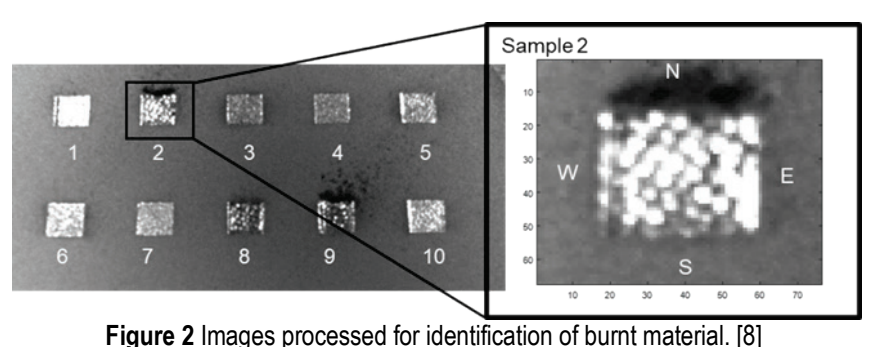


The four areas are selected corresponding to the hatch direction, as it has an influence on the position where the burnt material is deposited. In the current setup, in which Xand Y-hatches are used, the accumulation of burnt material is found either in the North or in the East area, depending on the layer.

For each of the areas, the grayscale distribution is calculated and presented in the form of a histogram. A curve fitting with a standard Gaussian curve is applied to each of the histograms, Fig. 3. These show different results for areas with and without burnt material. The widths of the curves differ, as represented by the coefficient $\mathrm{c}_{1}$. A larger value indicates a larger width. Furthermore, the goodness of fit is different, which is represented by the $R^{2}$ value. A larger value indicates a better fit of the curve.

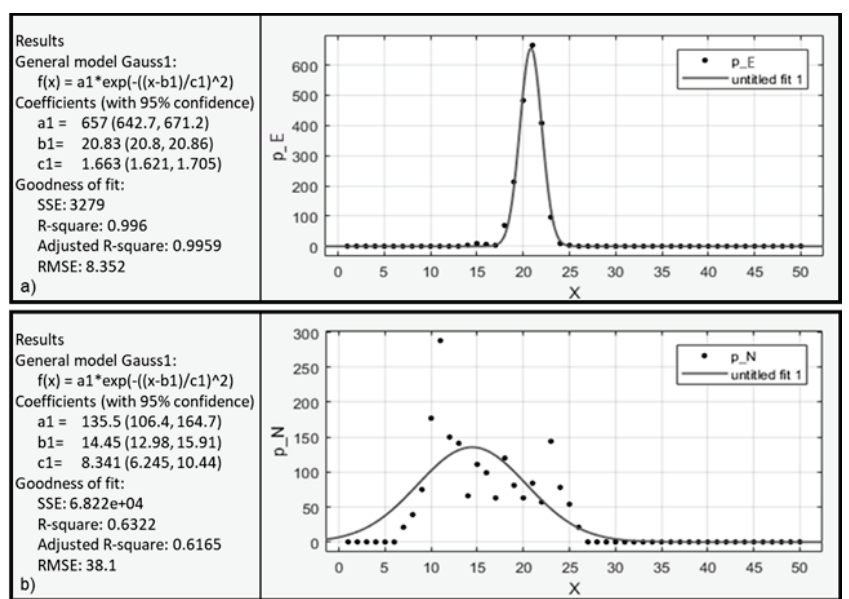

Figure 3 Grayscale distribution and fitting of a Gaussian curve for a) East and b) North of sample 2 [8]

By combining these to values an indicator $\mathrm{I}_{\mathrm{b}}$ for the presence of burnt material can be calculated according to Eq. (2)

$$
I_{\mathrm{b}}=\frac{c_{1}}{R^{2}}
$$

For the East area, where no burnt material is present, the indicator is $I_{\mathrm{bE}}=1.7$, whereas the indicator for the North area is $I_{\mathrm{bN}}=13.2$. A clear difference between the indicators is obvious. A further advantage of using a relative value as an indicator is that it filters the effect of different lighting situations in different areas of the build envelope, which can be seen in Fig. 2. These differences occur due to the arrangement of camera and light sources relative to the build platform, which is not concentric. Thus, a comparison of absolute values delivers results dependent on the part position.

\subsection{EXTENSION TOWARDS VARYING CROSS SECTIONS}

In chapter 3.2 the principal calculation method for the indicator is shown. The procedure is shown with the simple example of a cube that has edges parallel to the pixel matrix of the image. Furthermore, the cross sections are constant over the entire build height. This special situation makes it easy to evaluate the edges around the melted parts from the photo sections. However, if the components are more complex in their basic structure and the exposed areas change over the component height, this must be taken into account when determining the North, East, South and West areas to be evaluated. The scanning direction of the hatch in the respective layer is also to be considered.

The shape of the histogram of areas with burnt material depends not only on the amount of burnt material, but also on the size of the partial area considered in principle. If, for example, a very large partial area is analysed in which only a small area segment is covered with burnt material, this will hardly be noticeable in the histogram and in the evaluation proposed in chapter 3.2. Furthermore, observations have shown that small melted areas also result in smaller areas with burnt material [15]. This means that the size of the partial areas to be investigated has to be determined depending on the structure and shape of the component to be built.

The following procedure has been developed for this situation: The position and shape of the individual components per layer is known from the pre-process (Fig. 4).

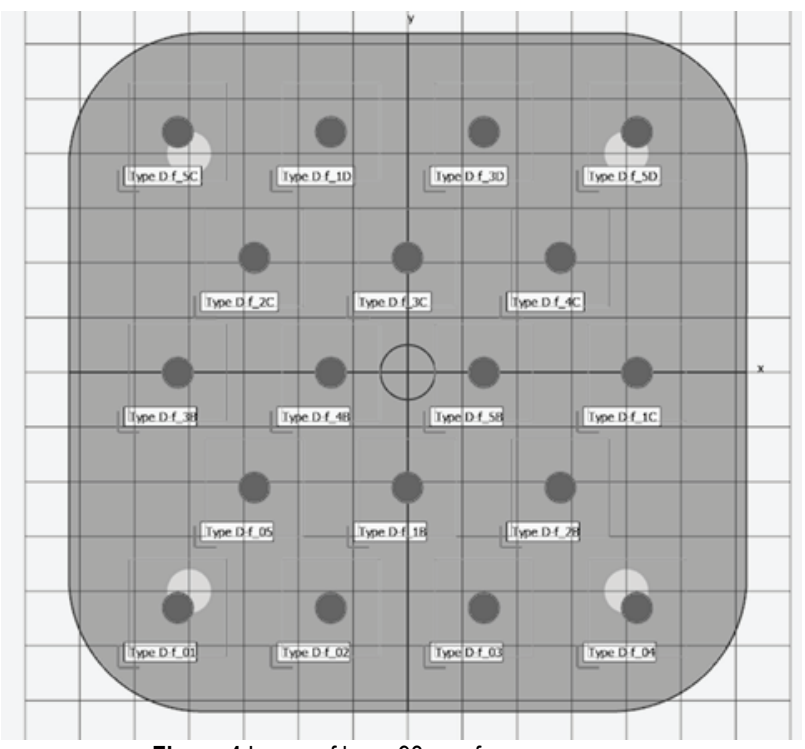

Figure 4 Image of layer $98 \mathrm{~mm}$ from pre-process.

Based on this information, the size of the partial area to be exposed can be determined. Classical image processing tools can automatically determine the size, position and shape of the partial areas to be exposed layer by layer. In addition, information about the hatch strategy can be obtained to identify relevant areas for evaluation.

Masks are created here from the pre-process images. If these masks are overlaid with the images of the build envelope, the actual components are faded out and thus only unexposed areas (powder bed) are visible for the grayscale analysis.

It should be noted that the images of the build envelope are in distorted perspective, as the camera is located not centered in the build envelope at a distance of $22 \mathrm{~cm}$ from the 
powder bed surface, while the images from the pre-process are generated by the computer and do not show any perspective distortion. In order to be able to overlay the generated masks with the image of the build envelope, the image distortions must be corrected by image transformations (Fig. 5).

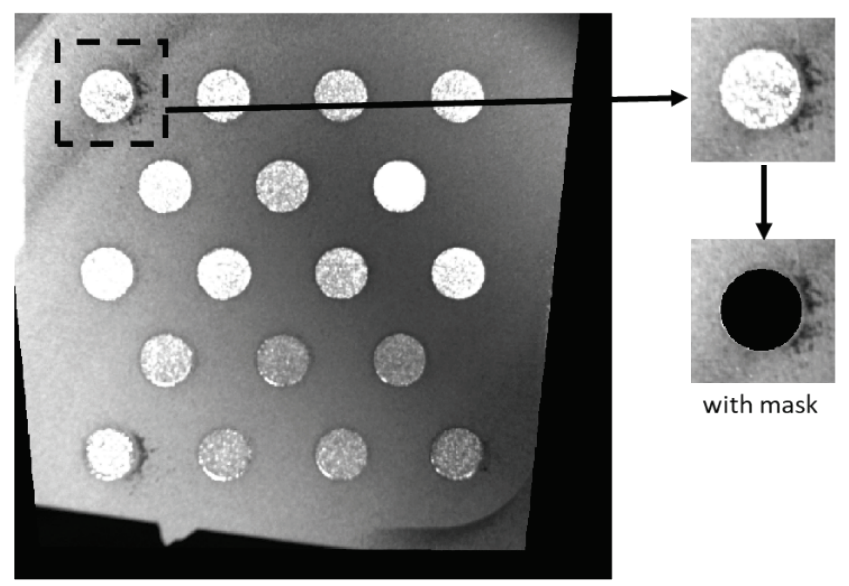

Figure 5 Transformed image a) Detail b) Detail with mask.

\subsection{Evaluation}

For evaluation, the developed method is applied to a successive build job. In this build job round tensile test specimen (Fig. 6) with conical clamping heads according to DIN 50125 - D $5 \times 25$ [14] with an increased parallel length of $80 \mathrm{~mm}$ (total length of $104 \mathrm{~mm}$, maximum clamping head diameter of $18 \mathrm{~mm}$ ) are built. The support in this build job is $4 \mathrm{~mm}$ high. Therefore, the overall height of the build job is $108 \mathrm{~mm}$.

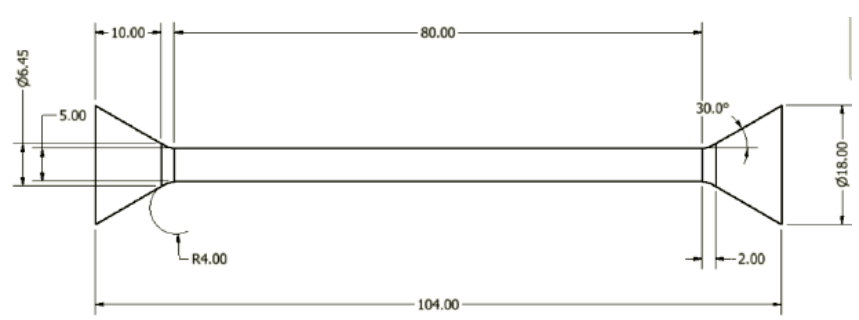

Figure 6 Tensile test specimen [9]

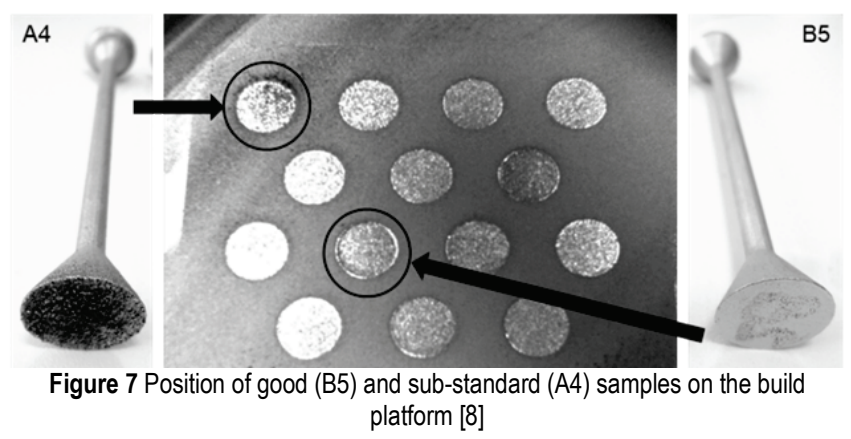

The evaluated images are captured during the production of the parallel section and the upper clamping head. Due to different parameter settings good and sub-standard parts are produced in this build job, as shown in Fig. 7.

An accumulation of burnt material is clearly visible in the area of the sample A4. Furthermore, the final part shows a rather strong deformation of the surface, resulting in a substandard quality of the part. In comparison to this, the sample B5 shows a smooth surface and no disturbances of the surrounding powder bed are visible during the build process.

The developed image processing method is applied to the images form the layer height $73 \mathrm{~mm}$ to $108 \mathrm{~mm}$, which includes 1070 successive layers. Fig. 7 shows the resulting indicator $I_{\mathrm{b}}$ for the analysed layers of the sample A4. The position of the deposited burnt material is depending on the hatch direction and changes between the layers with $\mathrm{X}$ - and Y-hatch. To enable an automated calculation of the indicator, it is calculated for all four regions in every layer. For further evaluation, the area with the lowest $R^{2}$ value is considered in each layer. Those values are represented by the graph in Fig. 8.

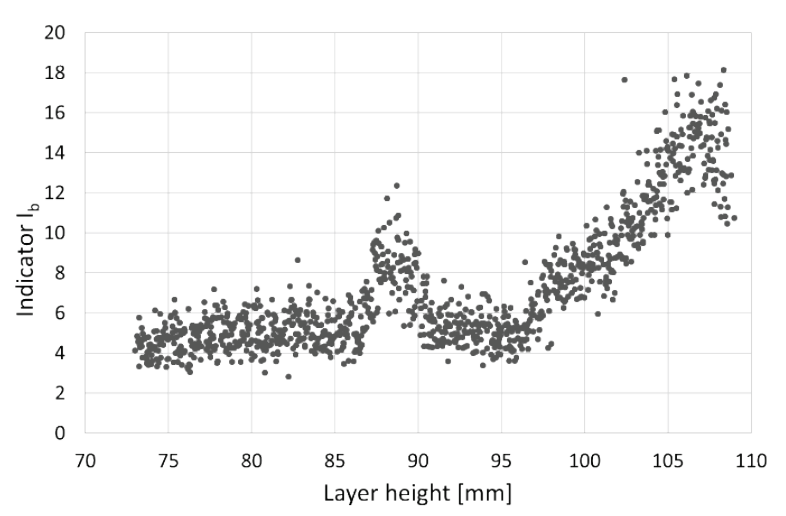

Figure 8 Indicator $I_{\mathrm{b}}$ of sample A4 (Layer height $73 \mathrm{~mm}$ to $108 \mathrm{~mm}$ )

From the diagram, it can be seen that the $I_{\mathrm{b}}$ value has a certain fluctuation margin, therefore a moving average over 50 layers is used for further evaluation. Fig. 8 shows the moving average of the indicator $I_{\mathrm{b}}$ for the samples A4 and B5 for the layer height from $73 \mathrm{~mm}$ to $108 \mathrm{~mm}$. With these signals, the changes during the production of the individual components can be easily observed.

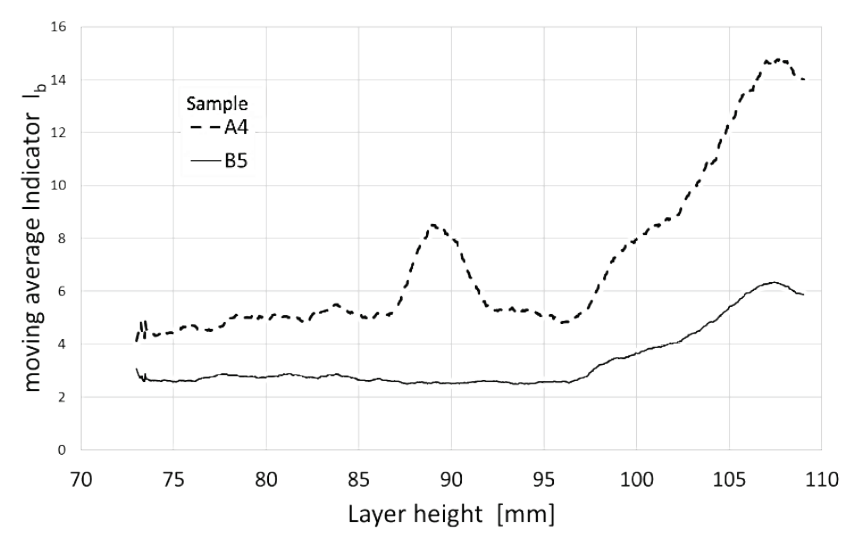

Figure 9 Moving average of indicator $I_{\mathrm{b}}$ of sample A4 (height $73 \mathrm{~mm}$ to $108 \mathrm{~mm}$ ) 
The comparison of the values shows a significantly different behaviour of the indicator values for the two samples (Fig. 9). The indicator for sample B5 is at a low level. There is an increase of the indicator from the height where the constant cross-section merges into the conic area. The indicator curve of sample A4, however, shows clear differences. The indicator value already starts at a higher level. At the layer height of $88 \mathrm{~mm}$, the value increases, reaches a local maximum and decreases again. In this range the cross-section of the tensile sample is still constant. By closer inspection of the images, a grey haze in the powder can be seen in the area of this sample (Fig. 10a and Fig. 10b). There is a clear second increase of the values in the area of the conic clamping head. Here, the cause is an accumulation of burnt material (Fig. 10d).

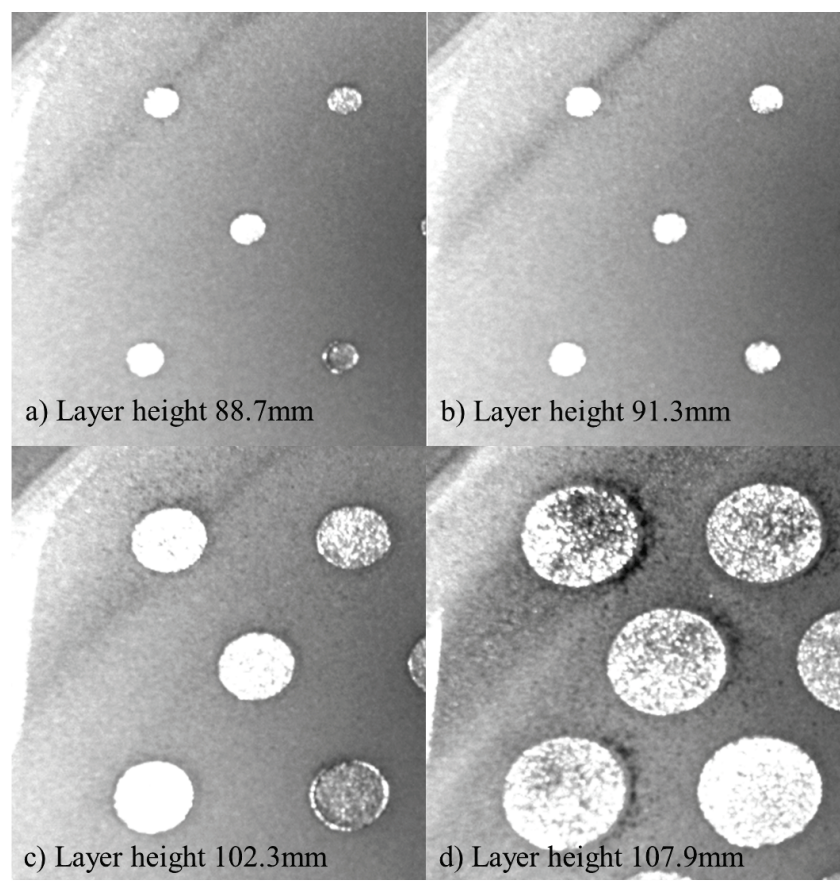

Figure 10 Captures of part A4 (top right) at different layers

This dissimilar behaviour of the different sections of the sample is likely to be caused by the different size of the exposed cross sections. While the inserted energy per square millimetre is the same, the overall amount is smaller due to the smaller cross-section in the layers representing the parallel length. Furthermore, the heat conduction is influenced not only by the exposed area in the layer itself but also by the volume of solidified material in the previous layers.

\section{CONCLUSION AND OUTLOOK}

The presented image processing method is currently applicable to identify parts which are likely to cause severe problems in successive layers. As only a limited area of the powder bed surface is analysed, applying standard procedures of image processing, the processing time is relatively short. Integrating this evaluation into the process monitoring tools of an M-LPBF machine can help to identify parts with problematic energy input before they start to grow out of the powder bed and may cause damages to the recoated system.

To define the areas for monitoring, it is necessary to know, which parts of the powder bed are exposed in each layer. For further reduction of the processing time, additional information on the direction of the hatch scan can be included. As all this information is available before the start of the build job, the areas of interest can be calculated for each layer in advance.

One of the next steps is to work out which signal changes lead to which actions in the build process. A first step could be to switch off individual problematic components and continue building the rest of the build job.

A further possibility is the local reduction of the energy introduced into a component in the next layers as soon as a possible fault is detected [7].

Investigations have shown that the volumetric energy density in relation to a component depends not only on the laser power but also on movement parameters of the laser [10]. In order to be able to reduce the energy introduced into the component, further investigations into the effects of the parameters of the volumetric energy density must be carried out.

\section{Acknowledgements}

The project "Contribution to quality assurance in additive manufacturing of individual products based on CoCr-alloys" is supported by the Hochschulgesellschaft OWL e.V.

The project "Measuring systems for quality management in additive manufacturing (MAQAM)" is funded by the $\mathrm{FH}$ Basis program of the Ministerium für Kultur und Wissenschaft des Landes Nordrhein-Westfalen.

Notice

The paper will be presented at MOTSP 2020 International Conference Management of Technology - Step to Sustainable Production, which will take place from $30^{\text {th }}$ September $-2^{\text {nd }}$ October 2020 in Bol, island Brač (Croatia). The paper will not be published anywhere else.

\section{REFERENCES}

[1] Acatech - Deutsche Akademie der Technikwissenschaften, Nationale Akademie der Wissenschaften Leopoldina, Union der Deutschen Akademien der Wissenschaften (Eds.) (2016). Additive Fertigung, München.

[2] Verein Deutscher Ingenieure (Ed.) (2014). Additive Fertigungsverfahren VDI Statusreport, Düsseldorf.

[3] Huxol, A. \& Villmer, F.-J. (2018). Process Control for Selective Laser Melting - Opportunities and Limitations, in: Villmer, F.-J., Padoano, E. (Eds.): Proceedings $8^{\text {th }}$ International Conference Production Engineering and Management, Lemgo, 17-28.

[4] Verein Deutscher Ingenieure (2014). VDI 3405 Additive manufacturing processes, rapid manufacturing - Basics, definitions, processes, Beuth Verlag, Berlin. 
[5] Huxol, A., Scheideler, E., \& Villmer, F.-J. (2017). Influencing Factors on Part Quality in Selective Laser Melting, in: Padoano, E., Villmer, F.-J. (Eds.): Proceedings $7^{\text {th }}$ International Conference Production Engineering and Management, Lemgo, 10-24.

[6] Huxol, A., Davis, A., Villmer, F.-J., \& Scheideler, E. (2017). Deployment of Process Capability Analysis for Single-Part Production, in: Padoano, E., Villmer, F.-J. (Eds.): Proceedings $7^{\text {th }}$ International Conference Production Engineering and Management, Lemgo, 13-24.

[7] Scheideler, E. M. \& Ahlemeyer-Stubbe, A. (2017). Quality Control of Additive Manufacturing Using Statisitcal Prediction Methods, in: Padoano, E., Villmer, F.-J. (Eds.): Proceedings $7^{\text {th }}$ International Conference Production Engineering and Management, Lemgo, 63-74.

[8] Huxol, A., Scheideler E. M., \& Villmer, F.-J., (2019). Image Processing for Process Monitoring in Additive Manufacturing, in: Padoano, E., Villmer, F.-J. (Eds.): Proceedings $9^{\text {th }}$ International Conference Production Engineering and Management, Trieste, 241-252.

[9] DIN 50125:2016-12. Prüfung metallischer Werkstoffe Zugproben, Beuth-Verlag, Berlin.

[10] Huxol, A., (2020). Beitrag zur Qualitätssicherung in der additiven Fertigung individueller Produkte aus CoCrLegierungen, Dr.-Ing. Dissertation, Universität Paderborn.

\section{Authors' contacts:}

Eva Maria Scheideler, Prof. Dr. - Ing. (Corresponding author) Department of Production Engineering, TH-OWL University of Applied Sciences and Arts, Campusallee 12, D 32657 Lemgo, Germany +4952617025267, eva.scheideler@th-owl.de

Andrea Huxol, Dr. -Ing.

Quality Assurance, Hora Holter Regelarmaturen GmbH \& Co. KG, Helleforthstraße 58-60, D 33758 Schloß Holte-Stukenbrock, Germany +49520789031063, AHuxol@hora.de 